\title{
Musikkultur und Musikpflege am polnisch-sächsischen Hof
}

\author{
Alina Żórawska-Witkowska
}

1 Alina Żórawska-Witkowska, Muzyka na dworze Augusta II w Warszawie [Musik am Hof Augusts II. in Warschau], Warszawa 1997; Alina Zórawska-Witkowska, Muzyka na polskim dworze Augusta III, cześć I. [Musik am polnischen Hof Augusts III., Erster Teil], Lublin 2012. Eben dort finden sich auch detaillierte Hinweise auf die genutzten Archiv- und $\mathrm{Mu}$ sikquellen und auch auf die ständig wachsende Gegenstandsliteratur.

2 Zdzisław Jachimecki, Muzyka polska od roku 1572-1795 [Die polnische Musik von dem Jahre 1572-1795], in: Polska, jej dzieje i kultura od czasów najdawniejszych do chwili obecnej [Polen, seine Geschichte und Kultur von den ältesten Zeiten bis zur Gegenwart], Bd. II, Warszawa 1927, S. 553.
Das Thema umfasst eine über 60-jährige Regentschaft zweier Herrscher, die gleichzeitig Kurfürsten von Sachsen und Könige von Polen waren. Sie mussten also nicht nur ihre Anwesenheit und ihr Herz zwischen zwei Staaten, die im Hinblick auf ihre politische, wirtschaftliche und religiöse Struktur grundverschieden waren, teilen, sondern sie mussten ebenfalls ihre unterschiedlichen kulturellen Traditionen aufeinander abstimmen.

Der polnische und der sächsische Hof hatten die meiste Zeit über getrennte organisatorische Strukturen und getrennte Finanzierungsquellen. Ich hatte bereits Gelegenheit, die detaillierten Ergebnisse langjähriger Forschungen, die ich vor allem im Sächsischen Hauptstaatsarchiv Dresden und in der Sächsischen Landesbibliothek - Staats- und Universitätsbibliothek Dresden betrieben habe, in zwei meiner Bücher ${ }^{1}$, sowie in vielfältigen Beiträgen zu präsentieren. Daher konzentriere ich mich hier darauf, die Ähnlichkeiten und Unterschiede im Musikmäzenatentum dieser Herrscher an ihrem polnischen Hof darzustellen.

Die Herrschaftszeit der Wettiner in der Rzeczpospolita, der Adelsrepublik Polen-Litauen, wurde lange Zeit als Periode des von ihnen verschuldeten kulturellen Verfalls in diesem Staat, schließlich zu jener Zeit einer der größten Staaten Europas, charakterisiert. Erst in der zweiten Hälfte des 20. Jahrhunderts begann dieses in schwarzen Farben gehaltene Bild - eine Folge sowohl der politisch-historischen Propaganda als auch nicht vorhandener vertiefter Studien - lebhaftere Farben anzunehmen, ja in jüngste Zeit verblüfft es geradezu durch seinen Farbreichtum und seine Farbintensität. Noch 1927 erklärte der phänomenale Musikwissenschaftler Zdzisław Jachimecki im Brustton der Überzeugung, dass ,es nicht unsere [d. h. der Polen] Aufgabe ist, musikalische Tatsachen, die sich zwar auf polnischem Territorium abgespielt haben, aber nicht dem
Geist polnischer Musiker entstammten, zu schildern, obwohl sie zweifellos einen Einfluss, sogar einen gewaltigen, auf die weitere Entwicklung der Musikkultur der Nation hatten" ${ }^{\text {" }}$. Erst aufgrund der nach dem Zweiten Weltkrieg von einem ständig wachsenden Kreis von Historikern verschiedener Fachgebiete intensiver betriebenen Forschungen haben wir begonnen, allmählich eine durchaus interessante Vergangenheit wiederzugewinnen und entdecken den wesentlichen Einfluss der Sachsenzeit auf das Zeitalter der Aufklärung unter Stanislaus August Poniatowski.

In der Herrschaftszeit Augusts II. und $\mathrm{Au}-$ gusts III. in der Adelsrepublik registriere ich einige grundsätzliche Ähnlichkeiten. Es sind: 1. Eine ähnlich lange Regierungszeit, nämlich rund 30 Jahre; beide Monarchen erlangten die Krone übrigens unter überaus widrigen Umständen und in einem politisch stark uneinheitlichen Umfeld.

2. Eine ähnlich lange Anwesenheit in Warschau. August II. verbrachte hier während seiner 19 Aufenthalte summa summarum über elf Jahre, wobei es lediglich sechsmal vorgekommen ist, dass er rund ein Jahr in der Stadt residierte, viermal von zehn bis sechs Monate, die übrigen Aufenthalte dauerten von vier Monaten bis sogar nur einige Tage. August III. kam seltener nach Warschau als sein Vater, nämlich zehnmal, aber die Bilanz seiner Anwesenheit in Polens Hauptstadt fällt ähnlich aus, denn sie umfasst insgesamt zwölf Jahre. Der Aufenthalt des Monarchen in den Jahren 1756-1763 umfasst mehr als die Hälfte jener kumulierten Zeit, was jedoch durch den Siebenjährigen Krieg in Sachsen erzwungen worden war. Früher, d. h. im Laufe von über 22 Jahren, in welche die neun Aufenthalte Augusts III. in Warschau fielen, verbrachte er in dieser Stadt nicht ganz fünf Jahre. Die hier in aller Kürze angeführte Statistik zeigt klar die Einstellung dieser Herrscher zu ihren Pflichten in der polnischen Adelsrepublik.

3. Beim königlichen Mäzenatentum Augusts II. und Augusts III. lassen sich zwei Phasen 
unterscheiden. Die erste Phase des Mäzenatentums Augusts II., die in die Jahre 16971702 fällt, bezeichne ich als euphorische Periode, die zweite Phase hingegen, die die Jahre 1716-1733 umfasst, definiere ich als pragmatische Periode. Im Fall Augusts III. identifiziere ich die erste Phase in den Jahren 1734-1754, als der König Warschau selten besuchte und den Bereich seines Mäzenatentums äußerst vorsichtig ausweitete. Die zweite Phase hingegen bildeten die Jahre des Siebenjährigen Kriegs, als der Monarch seine künstlerischen Kräfte in Warschau konzentrierte.

4. Ich erblicke auch Ähnlichkeiten bei der Organisierung des künstlerischen Personals, das dem sächsischen und dem polnischen Hof diente. Das von August II. in der zweiten Phase seiner Regentschaft etablierte Modell wurde mit entsprechenden Änderungen von seinem Sohn übernommen.

Im Musikmäzenatentum beider Herrscher treten natürlich auch grundlegende Unterschiede auf. Sie betreffen vor allem unterschiedliche Geschmäcker und künstlerische Präferenzen, denn beide lebten ja auch in stilistisch verschiedenen Epochen. Die Herrschaft Augusts II. fiel in die Endzeit des Barocks, August III. regierte dagegen in Zeiten des aufkeimenden Klassizismus, der von dem immer stärker dominierenden galanten Stil angekündigt wurde. Daher konzentriere ich mich hier auf die Organisation des Musikund Theaterpersonals, was einen guten Überblick sowohl über die erwähnten Ähnlichkeiten als auch die Unterschiede vermitteln sollte.

\section{August II.}

August II. zog in den ersten Jahren seiner Herrschaft alle seine Theater- und Musikkräfte in Warschau zusammen. Nach Ansicht des kürzlich verstorbenen Historikers Jacek Staszewski war dies ein Versuch, „einen polnisch-sächsischen Hof, sozusagen einen Wanderhof, der sich einmal in Polen, einmal in Sachsen aufhielt, zu schaffen, konzipiert als der herrlichste Hof Europas, als Hof des ersten Kurfürsten des Reiches und Königs der mächtigen Rzeczpospolita ${ }^{3}$. Die „Königlich Pohlnische Capelle” wurde als gemeinsame Kapelle für Polen und Sachsen allmählich zu einem großen, ambitiös geplanten und dabei multinationalen Ensemble. Im Frühjahr 1699 zählte sie 41 Personen. Das Ensemble wurde von zwei Kapellmeistern gemeinsam geleitet - dem Sachsen Johann Christoph
Schmidt und dem Polen Jacek Różycki. Darüber hinaus gehörten der Kapelle ein italienischer Dichter, acht Sänger - davon sechs italienische und zwei polnische, zwei Organisten - davon ein Pole, 26 Instrumentalisten, ein Kopist und ein Diener an. Im Ensemble überwogen Deutsche, vermutlich Sachsen, aber eine große Gruppe bildeten auch die ehemaligen Kapellisten des Königs Johann III. Sobieski, darüber hinaus auch Musiker aus Wien und Krakau. In den folgenden Jahren schrumpfte die Kapelle, denn auch die Zeiten waren nicht günstig für sie - Inter arma silent Musae. Im Endeffekt wurde sie im April 1707 formal aufgelöst. Doch gleich darauf organisierte August II. ein neues Ensemble für den Bedarf in Dresden, für Warschau begann er erst Ende 1715 „Notre Orchestre de Pologne” $\mathrm{zu}$ etablieren.

Vorerst jedoch hatte die „Königlich Pohlnische Capelle" allerhand Gelegenheiten, sich in Warschau mit ihren Talenten und Möglichkeiten zu profilieren. Vor allem erforderten verschiedene Gottesdienste einen musikalischen Rahmen, wenngleich der Monarch keinen besonderen religiösen Eifer an den Tag legte. Die Musik begleitete auch Theateraufführungen, denen allerdings brachte der König großes Interesse entgegen.

August II. holte für seine erste Warschauer Theatersaison von Februar bis Mai 1699 ein italienisches Ensemble der Commedia dell'arte unter der Leitung von Gennaro Sacco vom Hof des Herzogs Georg Wilhelm von Braunschweig-Lüneburg in Celle nach Polen. Die Truppe präsentierte sowohl italienische als auch italienisch-französische Komödien (genau wie am Pariser Théâtre Italien), sie schwang sich außerdem dazu auf, ein Gelegenheitsstück - ein der italienischen Oper nahe stehendes Divertimento teatrale - aufzuführen, nämlich „Latona in Delo“ mit dem Text von Pietro Francesco Da Silva und der Musik von Johann Christoph Schmidt.

Nach der Abreise des Ensembles von Sacco hielt sich die französische Truppe von Denis Nanteuil, eines auf Bühnen in Frankreich, den Niederlanden und Deutschland erfahrenen Schauspielers und Sängers, von Mai bis August 1699 in Warschau auf. Im Mai 1700 wiederum kam die in Den Haag aufgestellte französische Truppe von Jean de Fonpré nach Warschau und im Juni traf ein in Paris formiertes ca. 50köpfiges Ensemble der französischen Oper, das von Louis Deseschaliers geleitet wurde, hier ein. Die Anwesenheit der sehr kostspieligen französischen Oper am polnischen Hof war eine Sensation in Mitteleuropa, denn
3 Jacek Staszewski, Polacy w osiemnast wiecznym Dreźnie [Die Polen in achtzehnten Dresden], Wrocław 1986 , S. 70 . 
solche Künstler waren im Allgemeinen nicht außerhalb ihres Vaterlands tätig.

So hatte Warschau also seit dem Frühjahr 1700 eine große Künstlerschar zu Gast, nämlich ca. 50 Sänger und Tänzer, die der Oper angehörten, mindestens 16 französische Schauspieler, den aus Paris gekommenen, hervorragenden Tänzer Louis de Poitiers, eine ca. 40-köpfige Kapelle, eine Janitscharenkapelle, Trompeter und verschiedene andere Musiker. August II. hatte jedoch nicht die Entwicklung der politischen Lage vorausgesehen, denn erst im November konnte nach der Rückkehr des Königs vom Livland-Feldzug intensiv Theater gespielt werden. So wurde u. a. das Gelegenheitswerk Divertissement ,pour le Retour du Roi à Varsovie“ - Libretto von Marc-Antoine Legrand, $\mathrm{Mu}$ sik von Renaud, Choreografie von Louis de Poitiers - aufgeführt. Die französischen Opernkünstler waren auch im Karneval 1701 aktiv, aber die Wirren des Nordischen Kriegs verlangten einen immer größeren Einsatz Augusts II. Deshalb wurde das Ensemble im August desselben Jahres entlassen.

Das ursprüngliche Konzept des gemeinsamen polnisch-sächsischen Hofs verfiel, als August II. abdankte, doch auch nach der Wiederherstellung seiner Herrschaft in der Adelsrepublik wurde es nicht wieder aufgenommen. Jetzt heuerte der König nämlich die meisten Ensembles, die übrigens von der sächsischen General-Accis-Casse bezahlt wurden, in Dresden an. Es waren: 1. die „königlich-polnische und kurfürstlich-sächsische” Kapelle (seit 1707), 2. die französische Truppe „La Comédie et la Danse“ (seit 1708), 3. die italienische Truppe „comici italiani“ (seit 1716), 4. die italienische Oper (1717-1720 und 1726-1733), 5. das katholische Kapellknabeninstitut (seit 1707), 6. das Ensemble der protestantischen Musik (seit 1697), 7. die Gruppe der Hof-Trompeter und Pauker (seit 1707), 8. die Gruppe der Bock-Pfeifer, 9. die Gruppe der Jagdpfeifer (Waldhornisten). 1719, als in Dresden einen Monat lang die Hochzeit des Prinzen Friedrich August und der Erzherzogin Maria Josepha gefeiert wurde, betrugen die Vergütungen der 185 Künstler, die nur der Kapelle und dem Theater angehörten, über 100.000 Taler, und das aus diesem Anlass errichtete Opernhaus verschlang ca. 148.000 Taler. In der Aufstellung (Etat) der polnischen Generalkasse hingegen befanden sich seit 1716 nur zwei Ensembles - die polnische Kapelle und die Janitscharenkapelle - sowie der Komponist italienischer Musik Giovanni Alberto Ristori, und seit 1729 auch eine Gruppe Bock-Pfeifer. 1729 betrugen die Ausgaben aus der polni- schen Kasse für Musik und Theater kaum 7.967 Taler.

Die „Pohlnische Capelle” Augusts II., vom König „Notre Orchestre de Pologne” genannt, war Ende 1715 entstanden und bis 1732 umfasste ihr Etat zwölf Instrumentalisten, doch in den letzten Monaten der Herrschaft des Königs wurde sie auf 15 Künstler aufgestockt. Entgegen dem Namen der Kapelle war sie ausschließlich von Musikern aus dem Heiligen Römischen Reich besetzt, ihr Name hingegen war darauf zurückzuführen, dass die Kapelle vor allem den Bedarf des polnischen Hofs befriedigte, obwohl sie, wenn nötig, auch nach Dresden beordert wurde. An der Spitze der Kapelle stand der Konzertmeister und musikalische Leiter Heinrich Schulze, ein heute nicht näher bekannter Musiker. Aber unter den einfachen Mitgliedern befanden sich auch Künstler, die später zu europäischem Ruhm gelangten. Es waren der Oboist und Flötist Johann Joachim Quantz (1718-1724 im Ensemble) sowie der böhmische Violinist František Benda (17311733 im Ensemble), die sich später an den Hof Friedrichs II. banden.

Mit der polnischen Kapelle arbeiteten auch zwei in Dresden residierende Komponisten zusammen, nämlich Giovanni Alberto Ristori (vor seiner Ankunft 1716 am Hof Augusts II. hatte er erfolgreich in Venedig gewirkt) sowie Louis André (seit 1720 von der sächsischen Kasse bezahlt, vorher mit Brüssel verbunden).

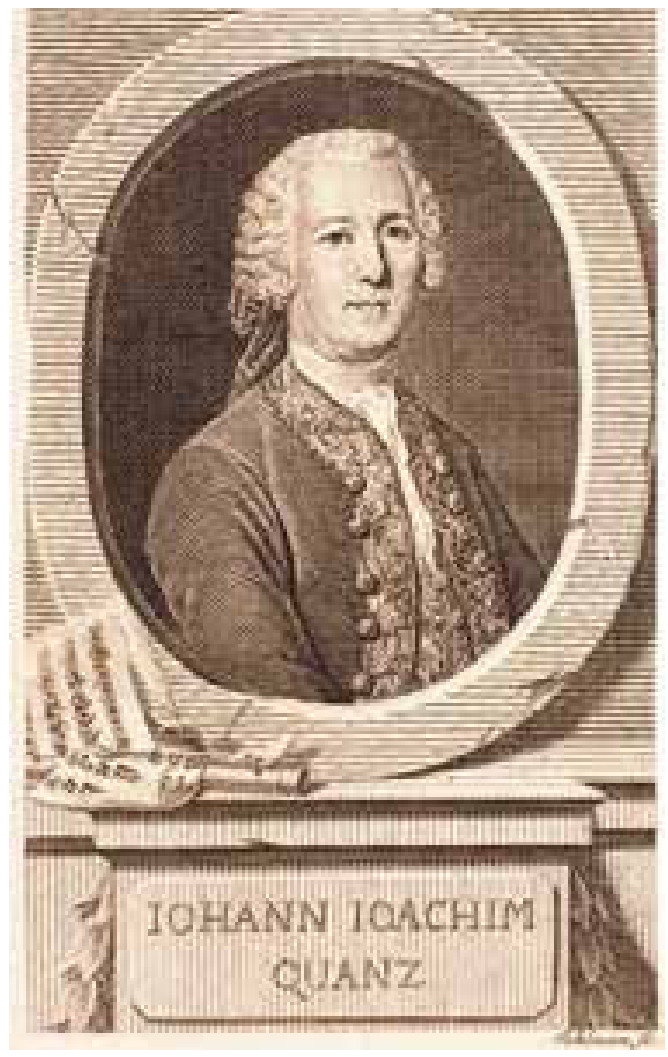

ponist Johann Joachim Quantz (1697-1773), welcher 1718 bis 1724 Mitglied der Königlich Kapelle von Starken war. Kupferstich Johann David Schleuen, um 1767 


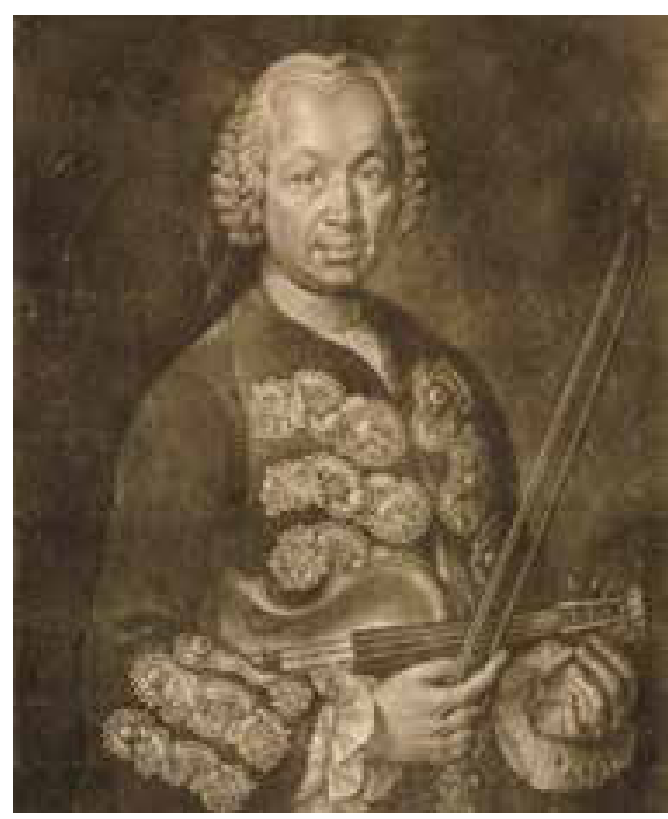

Sie wurden nach Polen beordert, je nachdem, ob der König Darbietungen seiner „comici italiani“ oder auch seiner französischen Truppe „La Comédie et la Danse“ in Warschau sehen wollte. Ein Vergleich der polnischen Kapelle Augusts II. mit seiner sächsischen Kapelle fällt natürlich zu Ungunsten ersterer aus, aber mit dem Dresdner Ensemble konnte sich damals wohl kein anderes in Europa messen, Quantz hatte jedenfalls nie ein besseres gehört.

Die Gruppen der Hoftrompeter und Pauker sowie der Bock-Pfeifer waren Formationen, die sowohl Sachsen als auch Polen dienten. Ihre Mitglieder waren allerdings Reichsbewohner. Die erste zählte zwölf Trompeter und zwei Pauker, die zweite 16 Bock-Pfeifer. Mit den Tönen der Trompeten und Pauken wurde den offiziellen Auftritten des Monarchen - seinem Eintreffen in der Stadt, Aufmärschen und Umzügen, feierlichen Gottesdiensten und Festmählern, der Begrüßung des Neuen Jahres und Ritterturnieren - Glanz verliehen. Bei ähnlichen Anlässen produzierten sich auch die Bock-Pfeifer. Die 24-köpfige Janitscharenkapelle hingegen, die an türkische Ensembles angelehnt war und auch solche Trachten trug, bestand aus $24 \mathrm{Mu}$ sikern mit polnischen Namen. Sie spielten auf türkischen Blasinstrumenten wie Sürmern und Schlaginstrumenten wie Pauken, Tellern und Tambours. Der exotische Klang solcher Kapellen rief bei den Zuhörern Verwunderung und Schrecken hervor. Selbst Jędrzej Kitowicz, ein Apologet der Sachsenzeit, musste einräumen, dass diese Musik „keinerlei musikalische Harmonie hatte, nur Quietschen und Rattern, aus der Ferne etwas angenehm, aus der Nähe schaurig" . August II. stellte seine Janitscharenkapelle auch auf internationalem Forum zur
Schau (Dresden 1719, Zeithain 1730), vermutlich zu dem Behuf, den Zuhörern seine vermeintliche Macht, die bis an die Grenzen des Osmanischen Reichs reichte, zu veranschaulichen.

Die ersten Spuren der Tätigkeit des 1709 in Dresden gegründeten königlichen französischen Ensembles „La Comédie et la Danse“ in Warschau stammen aus dem Karneval 1715, als es die Komödie „Le médecin malgré lui von Molière“ mit zwischengeschalteten musikalischen Intermedien aufführte. 1720 wurde aus dem Ensemble, das ein Jahr zuvor 21 Schauspieler, 23 Tänzer und vier Sänger gezählt hatte, die Hälfte seines Bestands sowie der Komponist Louis André nach Warschau abgezogen. Das Ensemble wurde damals in zwei Teile gegliedert, wovon einer für Polen und der andere für Sachsen bestimmt waren. Dieser Stand der Dinge währte bis 1724, als Warschau die Truppe ein letztes Mal zu Gast hatte. Die Zuschauer konnten damals das Können von Louis Dupré, einen der berühmtesten damaligen Tänzer, bewundern. Er war direkt aus Paris nach Warschau gekommen und hatte hier eine würdige Stütze in der Person von Jean Favier, des königlichen „maître de ballet“, gefunden.

Damals hielten sich die „comici italiani“ von Tommaso Ristori dreimal in Warschau auf, nämlich von Januar 1716 bis März 1717; von Oktober 1725 bis zum Herbst 1729 und von Oktober bis Dezember 1730. In Ristoris 19-köpfiger Truppe befanden sich auch Sänger, also enthielt das Repertoire außer der Commedia dell'arte ebenfalls italienische Intermezzi und Opern, hauptsächlich mit der Musik seines Sohns Giovanni Alberto Ristori. Erwähnenswert ist, dass französische Ballette in der Interpretation königlicher Tänzer in Begleitung von Jean-Baptiste Woulmier (Volumier), dem Konzertmeister der Dresdner Kapelle, der sich auf das Komponieren von Ballettmusik spezialisierte, in den Jahren 1725-26 die Warschauer Darbietungen der „comici italiani“ schmückten. Daher kann man hier von einem originellen Experiment, das zwei damals einander entgegengesetzte Musikstile, den italienischen und den französischen, miteinander verband, sprechen. Im September 1726 kehrten die Tänzer nach Dresden zurück und kamen während der Herrschaft Augusts II. nicht mehr nach Polen.

Im Herbst 1730 trafen hingegen die „comici italiani“, verstärkt durch Margherita und Cosimo Ermini, zwei Dresdner Sänger, die sich auf die Gattung der Intermezzi spezialisierten, in Warschau ein. Der Zweck des Erscheinens dieses Ensembles und einiger Musiker, die es begleiteten, bestand nämlich darin, dass es für ein
Der böhmische Geiger und Komponist František Benda (1709-1786), der von 1731-1733 zur Königlich polnischen Kapelle gehörte. Kupferstich von J. M. Schuster, um 1756
4 Jedrzej Kitowicz, Opis obyczajów za panowania Augusta III [Die Gewohnheiten während der Regierung Augusts III.], hrsg. von Roman Pollak, Wrocław 1970, S. 371. 
Jahr der Zarin Anna Iwanowna ausgeliehen wurde. Diese exotische Begegnung Italiens mit Russland und Russlands mit Italien verdient eine gesonderte Betrachtung. Jedenfalls kehrte Ristoris Truppe im Februar 1731 nach Warschau zurück, wo sie im April 1732 entlassen wurde. August II. war nicht mehr an ihrer Aktivität an seinem Hof interessiert - weder in Warschau, noch in Dresden.

\section{August III.}

August III. nutzte die von seinem Vater in der zweiten Phase seiner Regentschaft etablierten organisatorischen Modelle und platzierte seine Hauptmusik- und Theaterensembles ebenfalls in Dresden. Die sächsische Kasse bezahlte die Dresdner Kapelle „Die Königliche Capell- und Cammer-Musique”, das Ballett - „La Danse”, „andere Personen bey dem Theatro”, auch HofTrompeter und Pauker sowie Bock-Pfeifer, nicht mitgerechnet die Kirchenmusikensembles. Der Dresdner Kapelle gehörten mehrere Dutzend hervorragende Künstler an, nämlich der verantwortliche Leiter für das gesamte $\mathrm{Mu}-$ sik- und Theaterleben des Hofs, d. h. der „directeur des plaisirs“, der Kapellmeister, ein Dichter, Komponisten für Kirchenmusik, ein Komponist für Ballettmusik, Sänger, Instrumentalisten - darunter die Cammer-Musici, also Virtuosen, Kopisten, Organisten und Bedienteste. „Andere Personen bey dem Theatro” waren ein Inspektor, ein Baumeister, ein Bühnenmaschinist, Souffleure, Maler, Perückenmacher, Schneider, Tischler, Zimmerleute, Schlosser und dergleichen mehr. Aus der „Pohlnischen Reise-Cammer-Cassa“ wurden jedoch nur die Vergütungen der Mitglieder der polnischen Kapelle, die ihre Pflicht in Warschau tat, sowie der „comici italiani“, die genau wie unter August II. in Dresden residierten, bestritten. Die Pflichten der Mitglieder der Dresdner Kapelle, insbesondere des Leiters, des Dichters und der Komponisten, umfassten auch den Dienst am Warschauer Hof, denn in der Aufstellung der polnischen Kapelle wurde keines dieser Ämter erwähnt. Warschau verfügte auch nicht über eigenes technisches Theaterpersonal, sondern nutzte diesbezüglich die Fähigkeiten der Dresdner, die sicherlich von polnischen Kräften unterstützt wurden.

So also bildete Dresden wiederum das künstlerische Zentrum, aus dessen Bestand Warschau nur von Zeit zu Zeit, und das in begrenztem Maße, schöpfte. Hingen die Art und die Zahl der von Dresden nach Warschau beorderten Künstler doch von der politischen Situation, die Zeit und Dauer des Aufenthalts des Hofs im
Königreich bestimmte, ab. Diesbezüglich wurden jedes Mal andere Entscheidungen getroffen, daher unterschied sich jeder Aufenthalt Augusts III. in Polen im Hinblick auf das ihn begleitende künstlerische Personal.

Die polnische Kapelle Augusts III., die als „pohlnische Capelle”, „pohlnisches Orchester”, „das Orchester in Warschau”, „die Königl.[iche] Pohln.[nische] Capelle" bezeichnet und auf der Basis der polnischen Kapelle seines Vaters gegründet wurde, vergrößerte sich allmählich. 1753 erreichte sie einen 28-köpfigen Mitgliederstand und umfasste vier Sänger für die Interpretation von Kirchenmusik und 24 Instrumentalisten, die ein frühklassisches Standardorchester bildeten. In der Aufstellung der polnischen Kapelle (es sind 18 solche Listen aus den Jahren 1734-1762 überliefert) wurde niemals ihr Leiter erwähnt, lag doch die allgemeine Aufsicht über das gesamte Musikpersonal Augusts III. in den Händen des Oberkapellmeisters Johann Adolf Hasse, eines Komponisten, der damals in ganz Europa berühmt und geschätzt war. Wie er selbst erklärte, weilte er oft in Warschau und komponierte hier eine Reihe von Werken. Was die Besetzung des Ensembles im Hinblick auf die Nationalität seiner Mitglieder betrifft, so gehörten ihm trotz des Übergewichts der Deutschen (Sachsen?) auch Vertreter anderer Nationen an, wie Italiener, Böhmen, und was besonders wichtig ist, Polen - die Sänger Stefan Jaroszewicz (Kastrat), Józef Sękowski und Jan Stefanowski, die Organisten Piotr Kosmowski und Józef Czanczik, der Violinist Antoni Kossołowski vel Kozłowski (vom Hof des Hetmans Jan Klemens Branicki) und der Oboist Dominik Jaziomski.

Nach dem Tod Augusts III. fanden zehn seiner polnischen Kapellisten eine Anstellung im königlichen Orchester von Stanislaus August Poniatowski. Also hielt sich die langjährige Tradition der personellen Kontinuität in den Ensembles der aufeinander folgenden polnischen Könige. In der ersten Kapelle Augusts II. befanden sich Künstler Johanns III. Sobieski; den Grundstock der polnischen Kapelle Augusts III. bildeten Musiker seines Vaters; Stanislaus August Poniatowski gründete sein Orchester wiederum mit Instrumentalisten, die dem Ensemble Augusts III. angehört hatten.

Eine anregende Ergänzung der polnischen Kapelle Augusts III. war die 1735 in Warschau gegründete Kapelle des Ministers Heinrich von Brühl. Sie hatte nicht viele Mitglieder, dafür aber einige auserlesene Musiker, beispielsweise den Kapellmeister Gottlob Harrer, die Cembalisten Johann George Gebel und Johann Gottlieb Goldberg, den Violinisten Christian Fried- 
rich Horn, den Oboisten Johann Christian Fischer und den Violoncellisten Heinrich Megelin.

August III. griff in Polen auch auf Kirchenkapellen und Musiker zurück, die er von polnischen und litauischen Magnaten auslieh. Ständig unterhielt er darüber hinaus königlichkurfürstliche Hof-Trompeter und Pauker sowie Bock-Pfeifer, die jedes Mal aus in Dresden residierenden, gut ein Dutzend Musiker zählenden Ensembles ausgewählt wurden. August III. verzichtete dagegen darauf, die Janitscharenkapelle weiter zu behalten.

Die Opernsänger hatten die höchste Stellung in der Hierarchie der Musikberufe inne. Die Italiener beherrschten hier das Feld - allen voran die Kastraten. Während der Aufenthalte in Warschau half sich August III. diesbezüglich mit Sängern verschiedenster Provenienz aus. Gewöhnlich stammten sie aus seiner Dresdner Königlichen Capell- und Cammer-Music, mitunter gaben Sänger polnischer und litauischer Magnaten Gastvorstelllungen. Während des Siebenjährigen Kriegs tauchten Künstler auf, die der Hof hier mit dem Gedanken engagierte, sie später in Dresden einzusetzen. Jedenfalls wurden die Sänger in Warschau ad hoc ausgewählt - entsprechend dem geplanten Repertoire und den vorhandenen Möglichkeiten. Es ist nicht vorgekommen, dass sich während eines der Aufenthalte des Königs dieselbe Auswahl der Vokalisten wiederholt hätte.

Am Warschauer Hof Augusts III. gastierten im Laufe seiner Regentschaft ca. 30 Sänger von außerhalb der polnischen Kapelle. Die zahlreichste Gruppe bildeten die Kastraten - ca. 20 Sänger, darunter solche brillanten Künstler wie Domenico Annibali, Giovanni Bindi, Ventura Rochetti, Giuseppe Belli und Bartolomeo Putini. Es gab ca. zehn Künstler, hauptsächlich Deutsche, die über natürliche Stimmen (Tenöre und Bässe) verfügten. Noch dürftiger war die Repräsentation der Frauen, die aus kaum fünf, allerdings erstrangigen Künstlerinnen bestand, nämlich Margherita Delfini-Ermini, Caterina Pilaja, Teresa Albuzzi-Todeschini, Wilhelmine Denner und Elisabeth Teuber. Sie traten vor allem in italienischen Opern (opere serie), Gelegenheitsserenaden, Kantaten, Oratorien und Kirchenstücken, die während besonders feierlicher Gottesdienste aufgeführt wurden, auf. In Warschau wurden elf Opern, alle mit der Musik von Hasse, gespielt.

Die Truppe der „comici italiani“ Augusts III. entstand Anfang 1738 und hielt sich viermal in Warschau auf: von September 1738 bis Mitte Februar 1739, die folgenden Aufenthalte der Truppe in veränderter Besetzung fielen in die

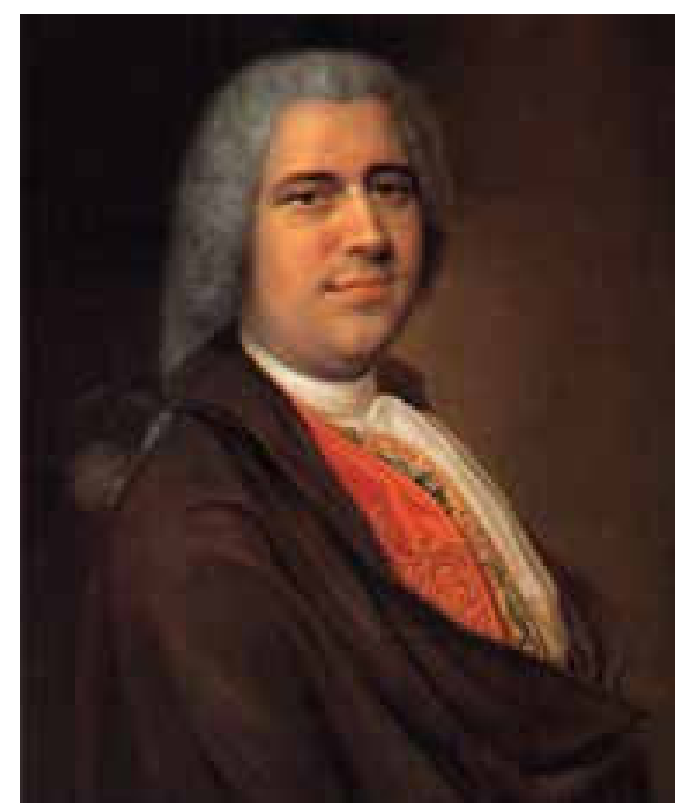

Der Komponist und sächsische Hofkapellmeister Johann Adolph Hasse (1699-1783), von welchem zahlreiche Opern in Warschau aufgeführt wurden. Gemälde von Balthasar Denner.
Jahre 1740, 1748/49 sowie in die zweite Hälfte des Jahres 1754. Eben die „comici italiani“ eröffneten am 3. August 1748 die Tätigkeit des neu erbauten Opernhauses und führten beispielsweise allein in der Saison 1748/49 33 italienische Komödien auf, sowohl dell'arte, als auch literarische. Dank solchen Schauspielern wie Giovanna Casanova (die Mutter des berühmten Verführers Giovanni Giacomo Casanova), Giovanni Camillo Canzachi, Cesare D’Arbes, Francesco Golinetti oder Marta Focari vel Focher, die früher in Venedig mit Carlo Goldoni verbunden waren, gelangte das Repertoire dieses exzellenten Lustspieldichters früh auf die Warschauer Bühne. 1754 trat ein Pole namens Muzierko mit der Truppe auf: Es war der Zwerg vom Hof des Fürsten Michał Fryderyk Czartoryski, Großkanzler von Litauen. Beachtenswert sind im Warschauer Repertoire der „comici italiani“ zwei satirische Opern, Parodien der Opera seria „Il Costantino“ (1739) sowie „Le contese di Mestre e Malghera per il trono“ (1748), deren Libretti wahrscheinlich Giovanna Casanova nach Werken von Antonio Gori verfasst hat. Im ersten Fall komponierte Giovanni Verocai die Musik und im zweiten Salvatore Apollini.

Die einzige Bastion des französischen Theaters am Hof Augusts III. war das Ballett, obwohl Italiener, Österreicher und Deutsche auch in dieser Materie immer nachhaltiger Fuß zu fassen begannen. August III. löste nach dem Tod seines Vaters die bisherige französische Truppe „La Comédie et la Danse“ auf, behielt jedoch eine Gruppe von Tänzern und gründete das Ballettensemble „La Danse“, das mit der Zeit auf 25 Künstler anwuchs. 1748 erschien in Warschau eine 16-köpfige Gruppe von Tän- 
zern, die aus dem Dresdner Ensemble ausgewählt worden waren, und die Darbietungen der „comici italiani“ begleiteten. 1754 hingegen trafen schon 29 Tänzer hier ein. Sie schmückten mit ihrer Kunst sowohl die italienischen Lustspiele als auch die Aufführungen der ersten Opera seria „L'eroe cinese“ mit dem Libretto von Pietro Metastasio und der Musik von Hasse. Unter den Balletttänzern fesselten Jean Favier, der „maître de ballet“, sowie die Solisten Antoine Pitrot, Caterina André und Jeanne Catherine Favier die Aufmerksamkeit des Publikums. Während des Siebenjährigen Kriegs verstreuten sich die Tänzer Augusts III. über ganz Europa, doch nach der Wiederaufnahme der Opernaufführungen in Warschau (1759-1763) kehrten sie allmählich in den Dienst des Königs zurück - u. a. Domenico Lenzi und seine Ehefrau Anette Tagliavini-Lenzi. Antoine Pitrot kam nach seiner Zeit der Zusammenarbeit mit den berühmtesten damaligen Ballettreformatoren - Franz Hilverding und Gasparo Angiolini als Künstler, der sich in den neuesten Trends bestens auskannte, zurück. Seine zwei Ballette, die am 3. August 1761 die polnische Premiere der Oper „L'Arminio“ - Libretto von Metastasio, Musik von Hasse - begleiteten, gehörten bereits der avantgardistischen Gattung „ballet d'action“ an.

\section{Musik- und Theatergeschmäcker Augusts II. und Augusts III.}

Am leichtesten lassen sie sich beim Theater identifizieren, denn hier sind die Quellen reichhaltiger und genauer. Die Warschauer Partituren zur Instrumental- und Kirchenmusik wurden nach dem Tod beider Herrscher den riesigen Dresdner Beständen einverleibt. Heute ist es schwer, sie dort aufzuspüren, d. h. ihnen eine Warschauer Provenienz zuzuweisen. Die Warschauer Theatersäle Augusts II., sowohl die im Königsschloss (nacheinander: das ehemalige Theater Ladislaus' IV. Wasa, das Theater im Alten Schloss, das Theater im Senatorensaal), als auch in der sächsischen Residenz (das Gartentheater, das Theater im Bokum-Palais, das Theater im Sächsischen Palais von 1724-1730) waren bezüglich ihres Baustils und ihrer Größe eher bescheiden. In den meisten war nur Platz für nicht viele - allerdings auserwählte - Zuschauer. Bescheiden sind auch unsere Kenntnisse von dem dort dargebotenen Repertoire, das zweifellos auf die frankophilen Neigungen des Monarchen hinwies. Die Truppe von Deseschaliers konnte im Schloss mindestens drei tragédies en musique von Jean-Baptiste Lully - „Armide, Thésée und Atys“ - aufgeführt haben. Später hingegen (1725/26) wurden bei- spielsweise Auszüge aus dem berühmten opéra-ballet „Les fêtés venitiennes“ mit der Musik von André Campry präsentiert. Unter den in Warschau gespielten französischen Komödien bildeten die mit Musik verzierten Werke wie comédie-ballets, comédies lyriques, Komödien mit Divertissements eine umfangreiche Gruppe. Selbst gesprochene Lustspiele wurden am Hof Augusts II. oft durch Ballettintermedien geschmückt. Jedenfalls waren das Werke, die eine Mischung aus Sprechdrama, Musik und Ballett bildeten, wie beispielsweise Molières „Le malade imaginaire“ (1722) oder „Le Bourgeois gentilhomme“ (1729).

Ein besonderer und untrennbarer Bestandteil des französischen Theaters war also das Ballett. Es gab jedoch damals auch schon Versuche, ein autonomes Ballett zu schaffen und die ersten zwei Beispiele bekam Warschau zu sehen. Es waren die in den 1720er Jahren aufgeführte symphonie de dance „Les caractères de la danse“ mit der Musik von Jean-Ferry Rebel sowie „Le ballet d'après Horace“ mit der Musik von Jean-Joseph Mouret.

August II. mied auch das italienische Theater nicht, wie ich bereits angedeutet habe, er bevorzugte jedoch leichtere Formen wie die Commedia dell'arte, musikalische Intermezzi, vermutlich auch die komischen Opern von Giovanni Alberto Ristori.

Als besonderes Verdienst für die „Rzeczpospolita" ist August II. anzurechnen, dass er in Europa, hauptsächlich in Deutschland, den sogenannten polnischen Stil, d. h. den Stil der polnischen Tanzmusik, lancierte. Es geht vor allem um die Polonaise, mit der der König u. a. am 4. September 1719 den großen Ball anlässlich der Hochzeit des Prinzen Friedrich August und der Erzherzogin Maria Josepha in Dresden eröffnete. August III. pflegte die Hofbälle mit der Polonaise zu beginnen. Seine sächsischen Nachfolger setzten diesen von ihm gefestigten Brauch bis 1918 fort. Die Arbeiten deutscher Musiktheoretiker, hier vor allem von Johann Mattheson und Johann Adolf Scheibe, wie auch die Werke vieler deutscher Komponisten, hier in erster Linie von Georg Philipp Telemann, Johann Sebastian Bach und Georg Friedrich Händel bestätigen nachdrücklich die Aufwertung der polnischen Tänze ${ }^{5}$.

So also gehörte der polnische Hof Augusts II. zu den interessantesten Musikzentren des damaligen Europa, umso mehr, als Warschau, selbst als Satellit Dresdens, ihm gegenüber bahnbrechende, direkt aus Paris (tragédie en musique, ballet d'action) oder aus Venedig (Intermezzi) übernommene Initiativen herausbrachte.

August III. vollzog im Bereich des Musiktheaters in Warschau eine Wende. Die ersten Büh- 
nen im Schloss $(1738 / 39)$ und in der sächsischen Residenz (1740) waren noch für einen nicht großen (80 bis ca. 150 Personen) und auserwählten Zuschauerkreis - kirchliche und weltliche Würdenträger der polnischen Adelsrepublik, Diplomaten, hochrangige Hofbeamte und Offiziere - bestimmt. Doch im Sommer 1748 eröffnete August III. im Sächsischen Garten die sog. Operalnia (das Opernhaus). Das Gebäude war von Carl Friedrich Pöppelmann entworfen worden. Die Baukosten betrugen etwas über 19.000 Taler (zur Erinnerung: Der Bau des Dresdner Opernhauses hatte fast 150.000 Taler verschlungen). Obwohl im Theater Plätze für ca. 570 Zuschauer vorgesehen waren, so verraten die überlieferten Listen der Personen, die Eintrittskarten (die vom Marschallsamt umsonst verteilt wurden) bezogen hatten, dass der Saal sogar 1.500 Personen fassen konnte, wenn diese sehr eng zusammenrückten. Natürlich erweiterte sich auf diese Weise der soziale Kreis der „spectateurs“, denn praktisch konnte jetzt jeder, der wollte, auch die Dienerschaft, ins Theater gehen. Eben im Opernhaus fanden ca. 120 Aufführungen zehn italienischer Opern Hasses, verziert durch zahlreiche Ballette, statt, darunter die Uraufführung der extra für Warschau komponierten Oper „La Zenobia“ (1761). In den Jahren 1761/62 nutzte eine Truppe der französischen Komödie unter Leitung eines gewissen Albani das königliche Opernhaus - ein vom Hof unabhängiges kommerzielles Ensemble, das gegen Entgelt (d. h. bezahlte Eintrittskarten) spielte. Die Rückkehr des französischen Theaters nach Warschau, das hier seit 1724 nicht mehr gesehen worden war, festigte den bereits strikt aufklärerischen Trend, der vom neuen König Stanislaus August Poniatowski entwickelt wurde. In den Jahren 1765-1767 nutzte Stanislaus August übrigens das vom sächsischen Hof gemietete Opernhaus, das zur Geburtsstätte des polnischen Nationaltheaters wurde.

Die meiste Zeit der Herrschaft Augusts III. konnte Warschau sowohl nach dem Willen des Monarchen, als auch mit Genehmigung der Rzeczpospolita in Bezug auf die Qualität des Musik- und Theaterlebens nicht mit Dresden mithalten. Immer jedoch belebte die Anwesenheit Augusts III. in Warschau die Stadt ungemein und machte sie zeitweilig zur künstlerischen Metropole Mittelosteuropas, die darüber hinaus stark mit den avantgardistischen Strömungen Westeuropas verbunden war. Allmählich lässt sich beobachten, dass August III. sich mehr und mehr um die künstlerischen Vorhaben des polnischen Hofs bemühte, als hätte er Vertrauen geschöpft in die Bürger der Adelsrepublik und sich von ihren kulturellen Aspirationen überzeugt. Doch erst während des Siebenjährigen Kriegs, insbesondere in seiner zweiten Phase (1759-1762), machte die damals ständige Anwesenheit des Monarchen in Warschau aus der Stadt ein Zentrum, das im Hinblick auf sein Musik- und Theaterleben mit Dresden, damals schließlich eines der hervorragendsten künstlerischen Zentren Europas, vergleichbar war. Die in Warschau aufgeführten und den hiesigen Bedingungen angepassten drammi per musica Hasses, die mitunter übrigens persönlich von ihrem Autor präsentiert und darüber hinaus durch die neuartigen ballets d'action in der Choreografie Pitrots verziert wurden, kann man zu den damals imposantesten europäischen Leistungen zählen.

Während des Kriegs weilte August III. länger in Warschau. Deshalb fokussierte er damals hier seine Aktivitäten als Kunstmäzen, die auch das geistige Leben der Stadt in Schwung brachten und außerdem das künstlerische Mäzenatentum der Magnaten der Adelsrepublik beflügelten. Einige von ihnen, übrigens Stammgäste des königlichen Opernhauses wie beispielsweise Jan Klemens Branicki, Michał Fryderyk Czartoryski, Michał Kazimierz Ogińnki, Franciszek Salezy Potocki, Michał Kazimierz Radziwiłł, Hieronim Florian Radziwiłł oder Wacław Rzewuski entfalteten an ihren Höfen ein interessantes Musik- und Theaterleben. Besondere Aufmerksamkeit verdienen solche Besucher der Warschauer Operalnia wie der spätere König Stanislaus August Poniatowski oder auch Ignacy Krasicki, der später nicht nur Bischof, sondern auch ein vortrefflicher Schriftsteller und in gewissem Grad sogar ein Musikmäzen war. Mit Sicherheit hatten die Erfahrungen aus dem Theater Augusts III. Einfluss auf ihre geistige Bildung gehabt. Im Repertoire seines königlichen Theaters widersetzte sich Stanislaus August Poniatowski den damals schon anachronistischen Geschmäckern Augusts III. und favorisierte modernere und dabei in der Perzeption für die Allgemeinheit leichtere Gattungen der italienischen Oper - die „opera buffa“, der französischen Oper - die „opéra comique“, der deutschen Oper - das Singspiel, ja sogar der polnischen Oper. Er rekrutierte jedoch sein königliches Orchester in erheblichem Maße aus Musikern, die früher der polnischen Kapelle Augusts III. und der Kapelle des Ministers von Brühl angehört hatten. Die Bereitwilligkeit dieser Musiker, in den Diensten des neuen Herrschers zu verbleiben, mag von der damals schon gefestigten Attraktivität Warschaus als Musik- und Theaterzentrum zeugen.

\section{Autorin}

Prof. Dr. Alina ŻórawskaWitkowska Uniwersytet Warszawski Instytut Muzykologii Krakowskie Przedmieście 32 00-325 Warszawa 